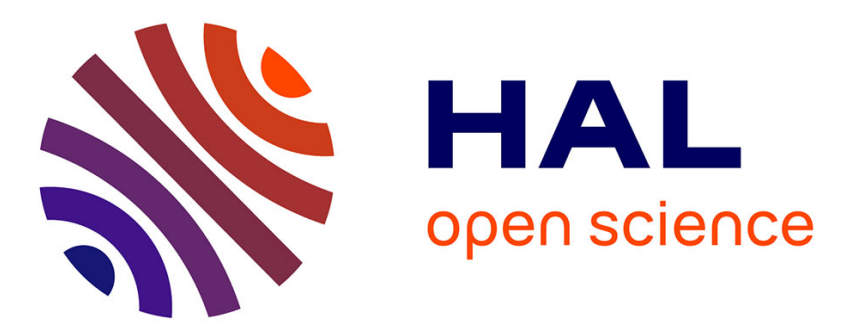

\title{
Treatment of human pancreatic cancer using combined ultrasound, microbubbles, and gemcitabine: a clinical case study
}

Spiros Kotopoulis, Georg Dimcevski, Odd Helge Gilja, Dag Hoem, Michiel Postema

\section{To cite this version:}

Spiros Kotopoulis, Georg Dimcevski, Odd Helge Gilja, Dag Hoem, Michiel Postema. Treatment of human pancreatic cancer using combined ultrasound, microbubbles, and gemcitabine: a clinical case study. Medical Physics, 2013, 40 (7), pp.072902. 10.1118/1.4808149 . hal-03193216

\section{HAL Id: hal-03193216 https://hal.science/hal-03193216}

Submitted on 16 Apr 2021

HAL is a multi-disciplinary open access archive for the deposit and dissemination of scientific research documents, whether they are published or not. The documents may come from teaching and research institutions in France or abroad, or from public or private research centers.
L'archive ouverte pluridisciplinaire HAL, est destinée au dépôt et à la diffusion de documents scientifiques de niveau recherche, publiés ou non, émanant des établissements d'enseignement et de recherche français ou étrangers, des laboratoires publics ou privés. 
1 Treatment of human pancreatic cancer using combined ultrasound,

2 microbubbles and gemcitabine: a clinical case study.

3 Spiros Kotopoulis $^{1,2}{ }^{*}$, Georg Dimcevski ${ }^{1,3}$, Odd Helge Gilja ${ }^{1,3}$, Dag Hoem ${ }^{4}$, Michiel

4 Postema $^{2,5}$

$5{ }^{1}$ National Centre for Ultrasound in Gastroenterology, Haukeland University Hospital, Bergen,

6 Norway

$7 \quad{ }^{2}$ Department of Physics and Technology, University of Bergen, Bergen, Norway

$8 \quad{ }^{3}$ Department of Clinical Medicine, University of Bergen, Bergen, Norway

$9{ }^{4}$ Department of Surgery, Haukeland University Hospital, Bergen, Norway

$10{ }^{5}$ The Michelsen Centre for Industrial Measurement Science and Technology, Bergen,

11 Norway

12

13 Abstract

14 Purpose: The purpose of this study was to investigate the ability and efficacy of 15 inducing sonoporation in a clinical setting, using commercially available technology,

16 to increase the patients' quality of life and extend the low ECOG performance grade;

17 as a result increasing the overall survival in patients with pancreatic adenocarcinoma.

18 Methods: Patients were treated using a customised configuration of a commercial

19 clinical ultrasound scanner over a time period of $31.5 \mathrm{~min}$ following standard 20 chemotherapy treatment with gemcitabine. SonoVue ${ }^{\circledR}$ ultrasound contrast agent was 21 injected intravascularly during the treatment with the aim to induce sonoporation.

22 Results: Using our custom acoustic settings, our patients were able to undergo an 23 increased number of treatment cycles; from an average of 9 to 16 cycles when

\footnotetext{
* Corresponding author email: Spiros.Kotopoulis@uib.no
} 
24 comparing to a historical control group of 80 patients. In two out of five patients

25 treated, the maximum tumour diameter was temporally decreased to $80 \pm 5 \%$ and

26 permanently to $70 \pm 5 \%$ of their original size, whilst the other patients showed reduced

27 growth.

28 We also explain and characterise the settings and acoustic output obtained from a

29 commercial clinical scanner used for combined ultrasound microbubble and

30 chemotherapy treatment.

31 Conclusion: It is possible to combine ultrasound, microbubbles, and chemotherapy in

32 a clinical setting using commercially available clinical ultrasound scanners to increase

33 the number of treatment cycles, prolonging the quality of life in patients with

34 pancreatic adenocarcinoma compared to chemotherapy alone. 
36 Cancer is the world's second largest cause of death with over 7.6 million deaths a year 37 (21\% of NCD deaths) [1]. There are over 217000 new cases of pancreatic cancer 38 worldwide every year [2]. Pancreatic cancer is very difficult to treat due to its aggressive biology, late diagnosis, the encasement of large blood vessels, and the presence of metastasis. Hence, surgery is rarely an option. Chemotherapy produces modest responses but is not curative in this setting, mainly because its use is severely hampered by toxic effects to vital organs. As a result, the survival is very low. The mortality of the inoperable patients is $50 \%$ within 3 months and $90 \%$ within 12 months $[3,4]$.

Sonoporation is a novel method for non-invasive targeted drug and gene delivery [58]. Sonoporation is defined as the transient formation of pores in cell membranes owing to ultrasound or a combination of ultrasound and microbubbles. These pores range in size from several nanometres to several micrometres [9-12], allowing for increased drug uptake in highly targeted regions [13-15].

The acoustic parameters used for sonoporation showing increased cellular uptake of

51 chemotherapeutics and genes vary from low-intensity diagnostic ultrasound 52 (Mechanical Index $(\mathrm{MI})<0.3)[16-29]$ to high-intensity diagnostic ultrasound $(\mathrm{MI}>$ 53 1.0) [9, 30-34]. Throughout literature, the acoustic settings used to induce 54 sonoporation vary drastically, with a broad range of these settings showing improved drug and gene delivery. Several studies also show the effect of clinical diagnostic

56 ultrasound in standard colour-Doppler and B-mode imaging on cellular uptake [19, 57 20]. These studies, which made use of clinical diagnostic scanners, concluded that a 58 larger duty cycle was necessary to increase the effect of sonoporation. It has been 
59 shown that the ideal settings to induce sonoporation are when shock-waves were not

60 present, in order to sustain the microbubbles, and when the duty cycle is long enough,

61 to excite the microbubbles in the targeted area without heating the surrounding tissue

62 [16]. Furthermore, higher intensities correlating to cavitation and jetting result in

63 increased cell death due to mechanical damage instead of (transient) sonoporation [33,

64 35-37]. As a result, there is no consensus on the exact ultrasound settings to be used

65 for sonoporation [38]. For this reason we aimed to use settings that matched our

66 previous in-vitro and in-vivo work as much as possible, i.e., an in-situ $\mathrm{MI}=0.2$,

67 maximum duty cycle, and minimum shockwave generation in order to preserve the 68 microbubbles $[7,8,23]$.

69 To date, all sonoporation experiments have been done either in vitro or in animal 70 models, hence the effect of sonoporation in humans is not truly known yet.

71 Ultrasound has been used as a tool in the clinic for many years, especially in 72 transabdominal imaging. Specifically, the pancreas can easily be imaged 73 ultrasonically [39]. In clinical ultrasonic imaging, ultrasound is combined with so74 called ultrasound contrast agents to locate tumours [40, 41]. These agents consist of 75 gas microbubbles encapsulated by elastic shells [42]. Using a clinical diagnostic 76 scanner for combined imaging and treatment allows for precise acoustic field 77 alignment ensuring that the correct ultrasound intensity reaches the target area.

78 In this study, we worked towards optimising the ultrasonic settings for invoking 79 sonoporation in the target region of a pancreatic tumour using a common commercial 80 clinical ultrasound scanner without physical modifications. 
83 A clinical scanner was calibrated in a degassed water bath in order to map the beam 84 profile and optimise the acoustic settings. After the chemotherapeutic dose was 85 delivered, the clinical probe was positioned aiming directly at the pancreatic tumour 86 and locked in place for 31.5 minutes. The probe was attached to a ball joint and was positioned near the upper abdomen. Stomach and intestine were avoided in all cases to ensure propagation only through soft tissue, to ensure delivery of the aimed ultrasound intensity at the desired area. Once the tumour was located the probe orientation was fine-tuned in order to locate the largest slice of the tumour and as much vasculature as possible, i.e., the feeding vessels. The probe was then locked in position until the completion of the treatment. The natural breathing motion aided the treatment as the ultrasound slice gently oscillated through the tumour. By visualising the vasculature and tumour it could be ensured that the microbubbles were being sonicated at the target. These vessels were then used as a reference point for future treatments. Nine doses of ultrasound contrast agent were intravenously injected over this time period to enhance the sonoporation effect. To evaluate the efficacy of the combined treatment we compared the amount of chemotherapy cycles the patient was able to receive. Furthermore, the tumour size was measured over the course of the treatment cycles to monitor and compare the tumour growth.

\section{Ultrasound scanner configuration}

104 A GE LOGIQ 9 ultrasound scanner (GE Healthcare, Waukesha, WI) combined with a

$1054 \mathrm{C}$ curvilinear probe (GE Healthcare) was used for both diagnosis and therapy. 
106 To calibrate and program the diagnostic scanner for the optimised therapeutic settings

107 the probe was locked in position in a custom-made 250-L 3D scanning tank,

108 containing degassed water. A calibrated HGL-200 bullet-type hydrophone (Onda,

109 Sunnyvale, CA) connected to a WaveJet 354a oscilloscope (Teledyne LeCroy SA,

110 Geneva, Switzerland) was used to measure the acoustic signal. The scanning tank had

111 a spatial resolution of $0.4 \mu \mathrm{m}$. For the calibration a $200-\mu \mathrm{m}$ resolution was used.

112 AQUASONIC® ultrasound transmission gel (Parker Laboratories, Fairfield, NJ) was

113 placed on the transducer transmission surface and the probe was subsequently covered

114 using a latex ultrasound probe cover (Sheathing Technologies, Inc., Morgan Hill, CA)

115 prior to submersion. The diagnostic scanner settings were modified in order to

116 achieve a maximum duty cycle without completely degrading the image quality, in

117 addition to having a linear acoustic signal. We aimed for minimal acoustic

118 shockwaves and harmonics minimising potential cavitation. The absence of nonlinear

119 content was verified by visualising the temporal extent of the pulses and performing a

120 Fast Fourier Transform (FFT) [43]. Multiple focal depths (from $2.8 \mathrm{~cm}$ to $8.4 \mathrm{~cm}$ ) and

121 different settings (varying gain, changing window size, etc.) were evaluated to ensure

122 similar acoustic conditions in all cases. To calculate the in-situ acoustic pressures and

123 intensities, the in-water values were derated by $0.3 \mathrm{~dB} / \mathrm{MHz} / \mathrm{cm}$, an approximation of

124 soft tissue attenuation in accordance to FDA and IEC guidelines $[44,45]$. The

125 attenuation factor of $0.3 \mathrm{~dB} / \mathrm{MHz} / \mathrm{cm}$ is only valid for soft tissue. Hence, this

126 calibration was representative for our clinical positioning for targeting the pancreas.

128 Table 1 shows the ultrasound scanner settings used to perform the simultaneous

129 observation and treatment of the pancreatic tumours. Skilled clinical sonographers

130 were called upon to judge the image quality. As there are variations between patients, 
131 such as tumour depth and tissue attenuation, certain settings had to be adjusted to

132 ensure the correct ultrasound intensity reached the required area whilst maintaining

133 the image quality. The settings that were varied are labelled as Patient-depending.

134 The three settings that were adjusted prior to treatment were: the focal depth, image

135 depth, and gain. The focal and image depths were adjusted in order to visualise and

136 position the acoustic focus directly in the middle of the tumour. By doing so we

137 could ensure that the acoustic conditions the tumour received was as similar as

138 possible in all patients. The gain is only applied after the received signal, hence it did

139 not affect the acoustic output. The gain simply allowed for a brighter image.

140 Once the probe was locked in position and the tumour was "targeted", no changes to

141 the ultrasonic conditions were made.

142

\begin{tabular}{|l|l|l|l|l|l|}
\hline & B-mode & Contrast mode & \multicolumn{3}{|c|}{} \\
\hline Parameter & \multicolumn{2}{|c|}{ Value } & Unit & Description & Variability \\
\hline MI & 0.4 & 0.4 & & Mechanical Index & None \\
\hline TIs & 0.0 & 0.0 & & $\begin{array}{l}\text { Thermal Index of soft } \\
\text { tissue }\end{array}$ & None \\
\hline Freq & 4.0 & 4.0 & MHz & $\begin{array}{l}\text { Centre Receive } \\
\text { frequency }\end{array}$ & None \\
\hline AO & 1 & 36 & $\%$ & $\begin{array}{l}\text { Normalised acoustic } \\
\text { output }\end{array}$ & None \\
\hline FR & 4 & 4 & fps & Frame rate & None \\
\hline Gn & $30-45$ & $30-45$ & $\mathrm{~dB}$ & Gain & Patient-depending \\
\hline S/A & $3 / 3$ & $2 / 0$ & & Synthetic Aperture & None \\
\hline Map & F/0 & $2 / 0$ & & Colour map & None \\
\hline F & $5.2-6.8$ & $5.2-6.8$ & $\mathrm{~cm}$ & Focal depth & Patient-depending \\
\hline D & $10-15$ & $10-15$ & $\mathrm{~cm}$ & Image Depth & Patient-depending \\
\hline DR & 66 & 66 & $\mathrm{~dB}$ & Dynamic Range & None \\
\hline SRI HD & 3 & 3 & & Image smoothing & None \\
\hline Grey Map & F/0 & $\mathrm{H}$ & & Image colour maps & None \\
\hline
\end{tabular}




\begin{tabular}{|l|l|l|l|l|l|}
\hline Trig & -0.25 & - & $\mathrm{S}$ & Trigger delay & None \\
\hline Tint Map & D & - & & Image colour maps & None \\
\hline Trig & - & $0-1$ & & Image triggering & None \\
\hline TAD & - & on & & True Agent Detection & None \\
\hline F.Average & - & 3 & frames & Frame averaging & None \\
\hline
\end{tabular}

144 Table 1: Parameters as indicated on a GE LOGIQ 9 clinical ultrasound scanner.

145 The settings chosen resulted in acoustic conditions shown in Table 2 and beam

146 profiles shown in Figure 1.

147

\begin{tabular}{|c|l|l|l|l|l|}
\hline & $\begin{array}{l}\text { Centre } \\
\text { frequency } \\
(\mathrm{MHz})\end{array}$ & $\begin{array}{l}\text { Duty cycle } \\
\mathbf{( \% )}\end{array}$ & $\begin{array}{l}\text { Mechanical } \\
\text { Index }\end{array}$ & $\begin{array}{l}\text { Acoustic } \\
\text { power ISATA } \\
\left(\mathrm{mW} / \mathrm{cm}^{2}\right)\end{array}$ & $\begin{array}{l}\text { Peak peak- } \\
\text { negative } \\
\text { acoustic } \\
\text { pressure } \\
(\mathrm{MPa})\end{array}$ \\
\hline $\begin{array}{c}\text { in-water } \\
\text { values at } 6.7 \\
\text { cm depth }\end{array}$ & 1.9 & $\begin{array}{l}1(4 \text { cycles } \\
\text { every 0.21 } \\
\text { ms })\end{array}$ & 0.49 & 0.59 & 0.41 \\
\hline $\begin{array}{c}\text { Derated } \text { in- } \\
\text { situ } \text { values } \\
\text { at } 6.7 \mathrm{~cm} \\
\text { depth }\end{array}$ & 1.9 & $\begin{array}{l}1(4 \text { cycles } \\
\text { every 0.21 } \\
\text { ms })\end{array}$ & 0.20 & 0.25 & 0.27 \\
\hline
\end{tabular}

148

149 Table 2: Acoustic conditions generated by the 4C probe for sonoporation in-water

150 and derated for in-situ values [44, 45]. 

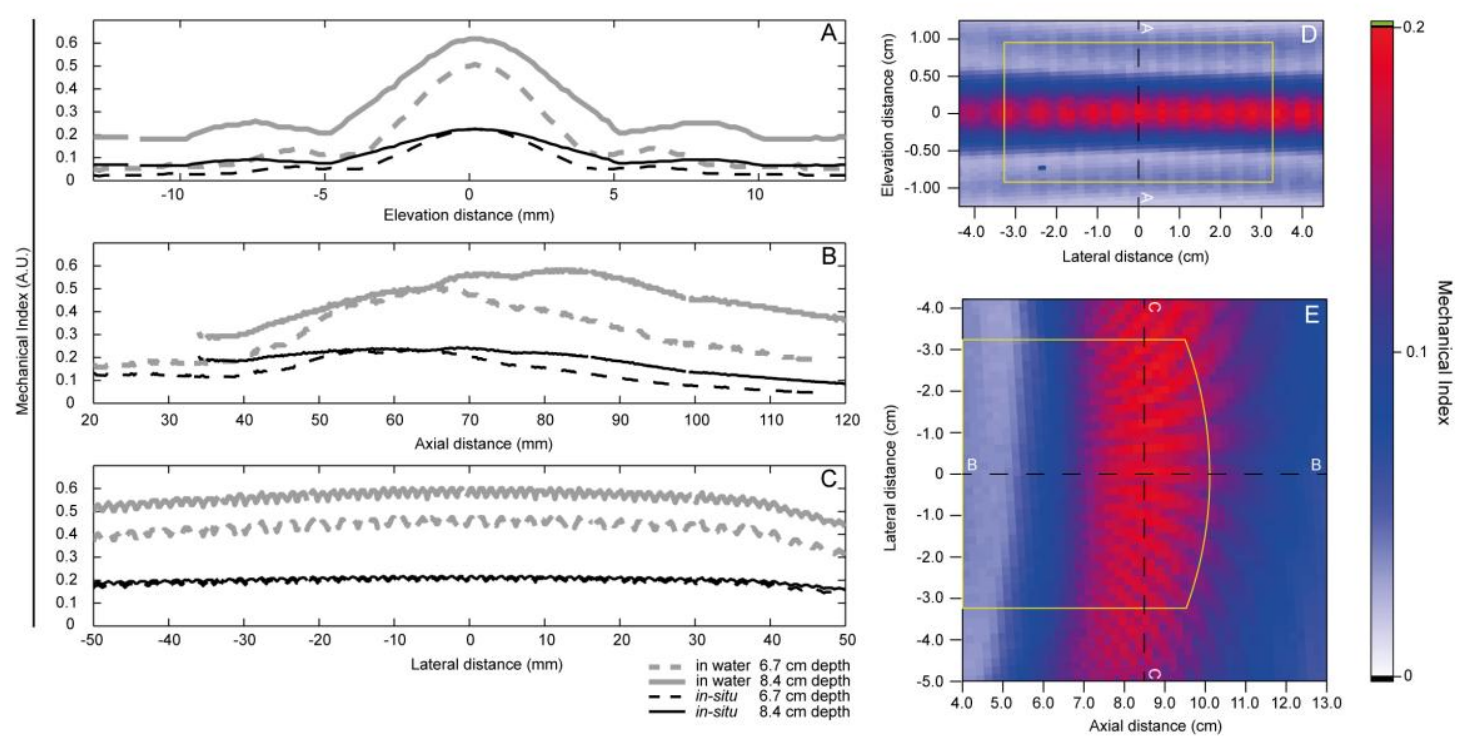

153 Figure 1: $1 \mathrm{D}$ and 2D beam profiles at sonoporation settings using the $4 \mathrm{C}$ probe at two

154 focal depths: $6.7 \mathrm{~cm}$ and $8.4 \mathrm{~cm}$ for the $1 \mathrm{D}$ plots and $8.4 \mathrm{~cm}$ for the $2 \mathrm{D}$ plots. The 155 beam profile was characterised in water and derated for in-situ values [44, 45]. Lines

156 A-A, B-B, and $\mathrm{C}-\mathrm{C}$ in panels $\mathrm{D}$ and $\mathrm{E}$ represent the position of the $1 \mathrm{D}$ scans shown in 157 panels $\mathrm{A}, \mathrm{B}$ and $\mathrm{C}$ respectively. The yellow bounding boxes in panels $\mathrm{D}$ and $\mathrm{E}$ 158 represent the area visible on the clinical scanner screen. In the elevation direction the 159 bounding box was defined by when a $0.5 \mathrm{~mm}$ needle could not be distinguished on 160 screen. The tumour was positioned at the intersection of lines B-B and C-C in frame E, 161 and at an elevation distance of $0 \mathrm{~mm}$ in frame $\mathrm{D}$.

163 The beam profile showed formation of multiple foci in close proximity along the 164 lateral direction merging to form a quasi-continuous focus (Figure 1E). In the 165 elevation direction side lobes can be clearly seen (Figure 1A and 1D). Using the full 166 width half maximum (FWHM) to define the beam size, the active or treatment area 167 can be defined as a volume of $69 \times>100 \times 1.0(\mathrm{~mm})^{3}(1 \times \mathrm{W} \times \mathrm{h})$. It is assumed that this 168 is the region were sonoporation occurred most efficiently. Figure 2 shows the pulse 
169 repetition pattern generated by these settings. The pulse was amplitude-modulated, 170 consisting of 5 cycles $(2.1 \mu \mathrm{s})$ every $210 \mu$ s corresponding to a $1 \%$ duty cycle

171 (repetition rate optimised). The duty cycle is defined as the percentage of time that

172 ultrasound is being generated. This was measured during the spatial calibration

173 process, in the acoustic focus with the hydrophone, for the duration of the inverse of

174 the frame rate. Due to synthetic aperture and contrast enhanced imaging the pulse 175 pattern at the focus was amplitude-modulated [46] [47]. This can be seen in the upper

176 panel of Fig. 2. The lower panel of Fig. 2 shows the time signal of a single pulse. The

177 pulse is still relatively sine-shaped, thus the transfer function of the propagation path

178 is linear. Minor nonlinear effects can be seen after the $4^{\text {th }}$ cycle. This indicates that 179 shockwave occurrence and therefore microbubble destruction is negligible. 

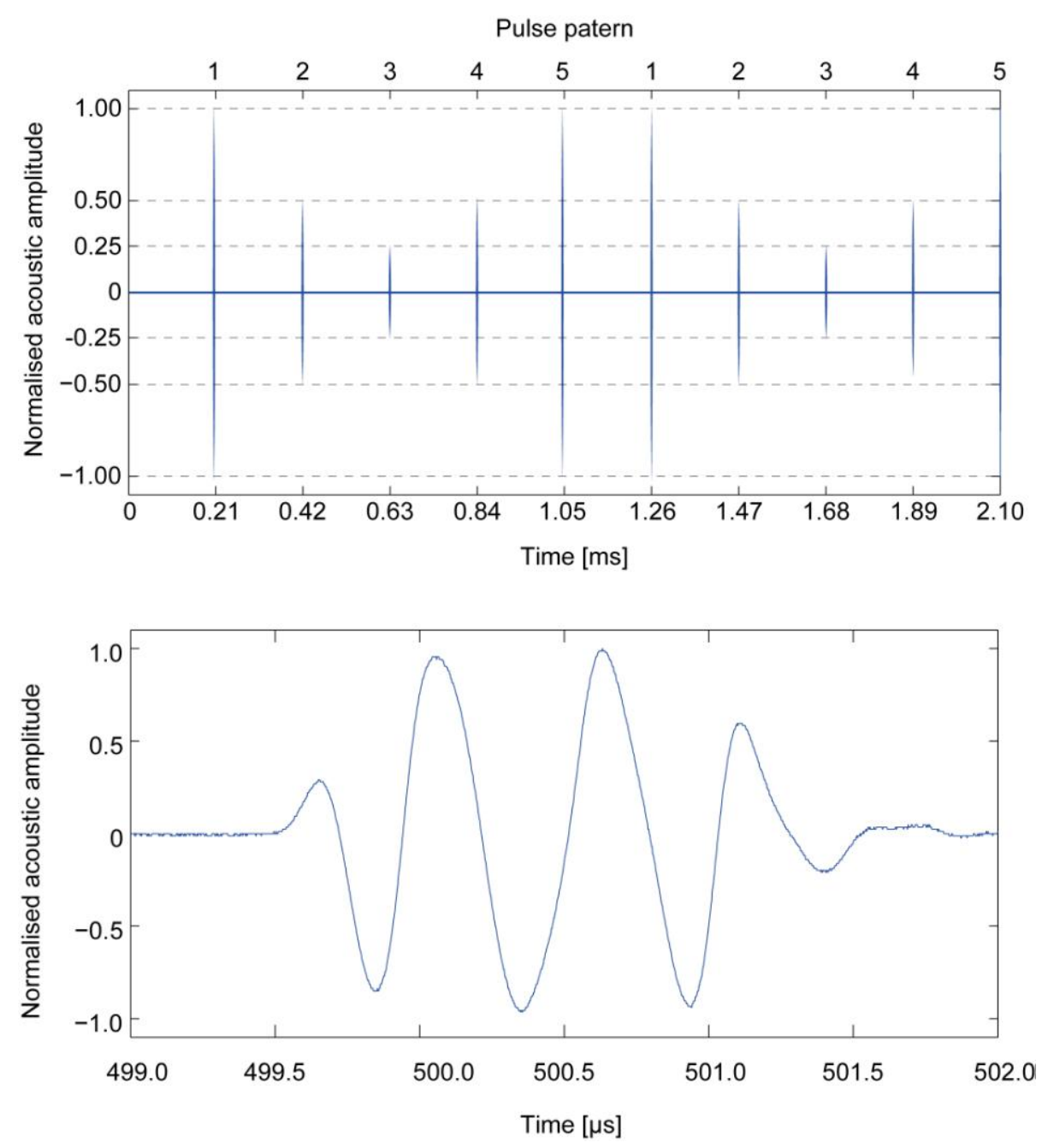

181 Figure 2: Ultrasonic pulse generated by the clinical scanner. The top panel shows the 182 pulse repetition frequency and pattern. The lower panel shows the temporal extent of 183 the pulse with the largest amplitude. The pulses were amplitude-modulated. Each 184 pulse consisted of 4 cycles $(2.1 \mu \mathrm{s})$ every $210 \mu \mathrm{s}$.

185 A Fast Fourier Transform (FFT) of the acoustic signal is shown in Fig. 3. The centre 186 frequency is $1.9 \mathrm{MHz}$. Using a $-3-\mathrm{dB}$ or FWHM cut-off the bandwidth was measured 187 to be $1.1 \mathrm{MHz}$; from $1.3-2.4 \mathrm{MHz}$. A second harmonic peak can be seen at $3.6 \mathrm{MHz}$ 188 due to the minor non-linear effects. This peak was $11 \mathrm{~dB}$ lower than the primary peak. 189 
190 These settings complied with current safety guidelines for clinical diagnostic imaging

$191[44,48,49]$. Figure 4 shows two images of pancreatic cancer in two separate patients

192 captured using the sonoporation treatment settings.

193

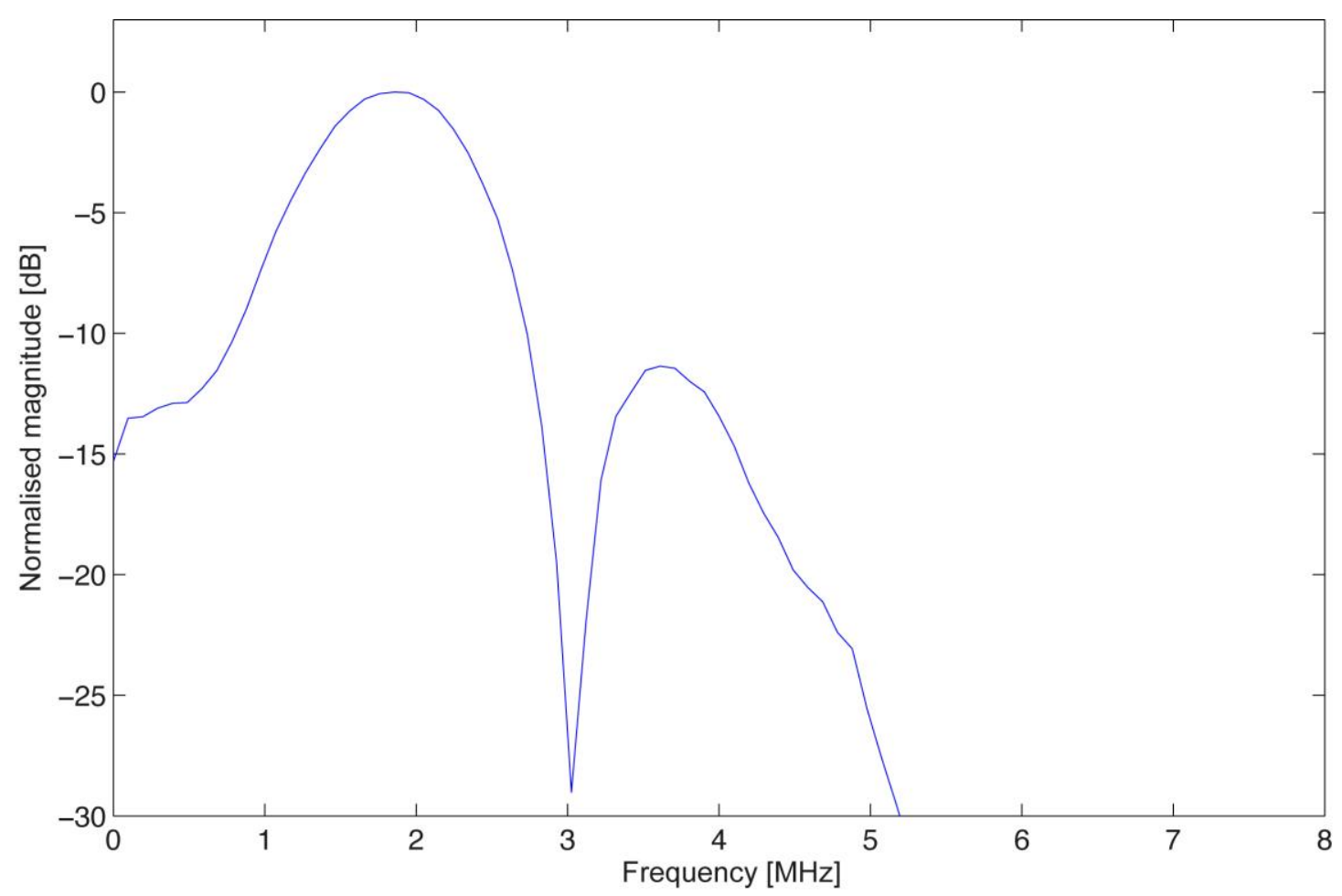

194

195 Figure 3: Fast Fourier transform of ultrasonic signal. The centre frequency of the

196 transmitted signal is $1.9 \mathrm{MHz}$. A bandwidth of $1.1 \mathrm{MHz}$ can be seen.

197
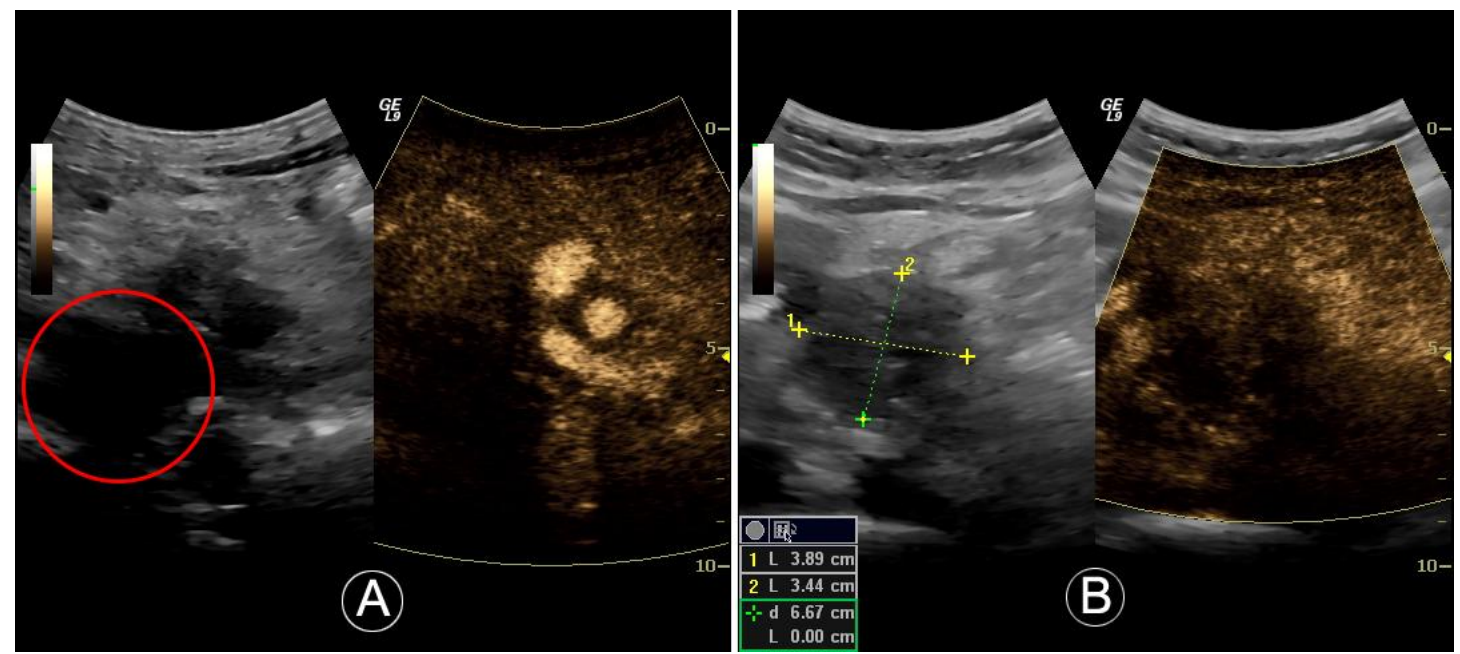
199 Figure 4: Images captured using customised sonoporation settings using a clinical 200 ultrasound scanner. The dense vasculature in early arterial phase to the right of the 201 main tumour (circled in red) can be seen in panel A. Panel B shows the dimensions of 202 the main tumour, indicated by lines 1 and 2, using the sonoporation settings.

\section{Chemotherapeutic and Microbubble dosage}

The recommended chemotherapeutic protocol was followed [50]. This protocol dictates which patients are eligible for chemotherapy and the dosages that can be administered. It includes dosage reduction values depending on platelet and absolute

209 granulocyte count. The chemotherapeutic used, gemcitabine (Gemzar ${ }^{\circledR}$, Eli Lilly and 210 Company, Indianapolis, IN) was administered once weekly for up to 7 weeks (or until 211 toxicity necessitates reducing or holding a dose), followed by a week of rest from 212 treatment. Subsequent cycles consisted of infusions once weekly for 3 consecutive 213 weeks out of every 4 weeks. Our protocol used the Eastern Cooperative Oncology 214 Group (ECOG) performance status as a measure of the clinical condition [51]. The 215 ECOG performance status ranges from $0-5$, where 0 denotes a "fully active patient 216 able to carry on all pre-disease performance without restriction", and 5 denotes a 217 "dead" patient. Chemotherapy was halted if the patient exceeded a grade of 2 that 218 states the patient is "ambulatory and capable of all self-care but unable to carry out 219 any works activities. Up and about more than 50\% of waking hours." The ECOG 220 guidelines can be considered as a measure of how "healthy" a patient is. We used the 221 ECOG guidelines to monitor the effectiveness of the combined treatment i.e., the 222 longer a patient stays below an ECOG grade of 3, the more effective the treatment is considered. 
224 A single treatment cycle is defined as a single infusion of chemotherapeutic followed

225 by ultrasound and microbubble treatment. The week pause was not counted as a 226 treatment cycle. Once the granulocyte or platelet count was permanently too low, or 227 the patient surpassed an ECOG performance status grade of 2, no more treatment was 228 administered.

230 Gemcitabine was administered by intravenous infusion at a dose of $1000 \mathrm{mg} / \mathrm{m}^{2}$ over 23130 minutes. The start of the chemotherapeutic delivery is defined as $T=0 \mathrm{~min}$. 232 During the last 10 minutes $(T=20 \mathrm{~min})$ of chemotherapeutic delivery, diagnostic 233 imaging was performed in standard abdominal imaging mode and the tumour was 234 located. Here the tumour dimensions were measured with ultrasonography. Once the 235 tumour was located, a custom made clamp was used to lock the probe in position and 236 the clinical scanner was switched to therapeutic settings (Fig 5). As the maximum 237 systemic concentration of the chemotherapeutic starts at the finish of delivery $238(T=30 \mathrm{~min})$ this was chosen as the initiation point for the ultrasound treatment. 239 Clinically approved SonoVue ${ }^{\circledR}$ (Bracco Imaging Scandinavia AB, Oslo, Norway) 240 ultrasound contrast agent was used as the microbubble for sonoporation. To ensure 241 microbubbles were present throughout the whole treatment $0.5 \mathrm{~mL}$ of contrast agent 242 followed by $5 \mathrm{~mL}$ saline were injected every $3.5 \mathrm{~min}$, i.e., at $243 T=30.0,33.5,37.0,40.5,44.0,47.5,51.0,54.5$ and $58.0 \mathrm{~min}$. A single vial $(4.5$ $244 \mathrm{~mL}$ ) was used throughout each treatment. Treatment was stopped at $T=61.5 \mathrm{~min}$. 245 The total cumulated ultrasound treatment time was only $18.9 \mathrm{~s}$. This time frame can 246 be seen in Fig. 5A. 
Gemcitabine infusion

Diagnostic scanning

A

Ultrasound and microbubble treatment

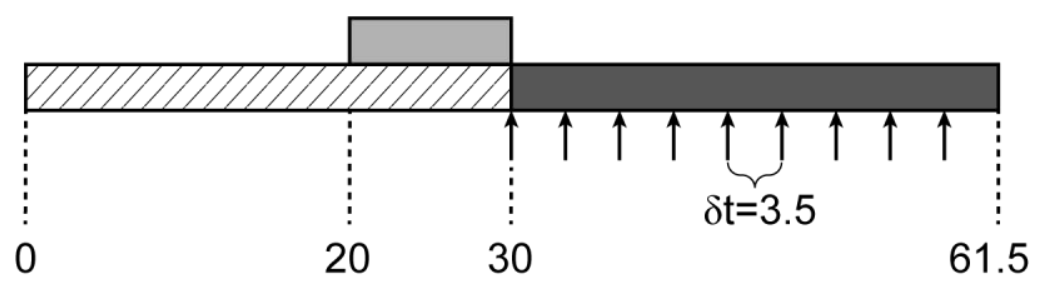

Time (min)

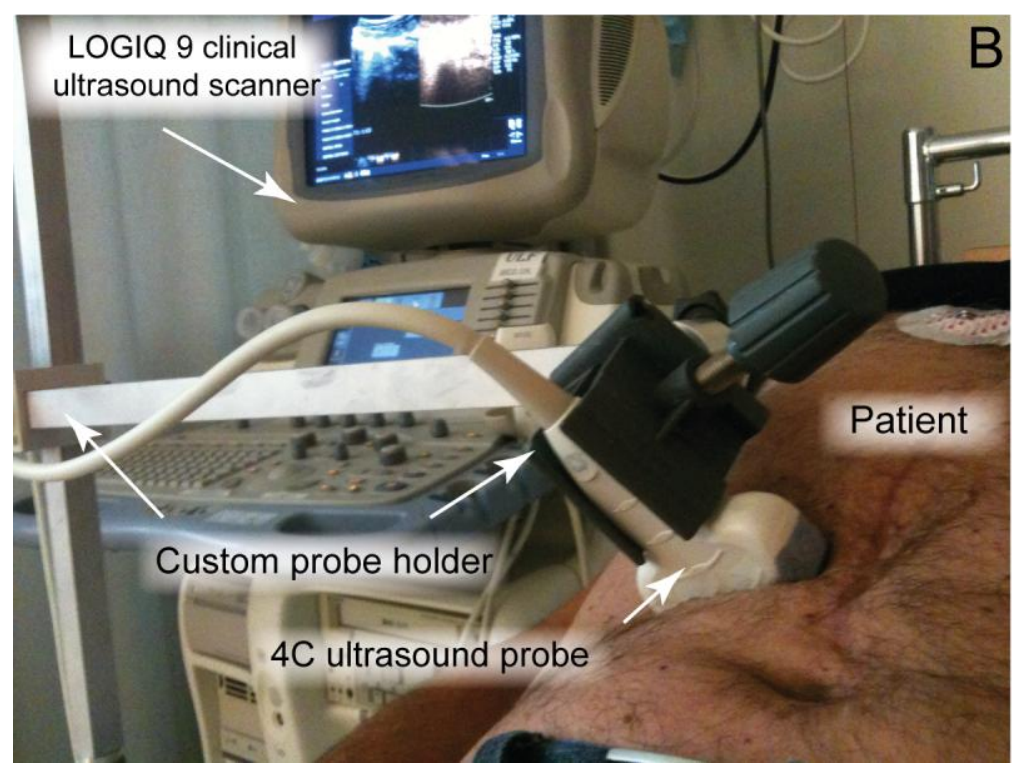

249 Figure 5: Time frame of each chemotherapy cycle (Panel A) and photograph of probe

250 and custom made probe holder during patient treatment using microbubble

251 sonoporation for pancreatic cancer (Panel B). Panel A shows the time frame for each

252 treatment cycle from the start of the gemcitabine infusion. Arrows indicate

253 intravenous injection time of $0.5 \mathrm{~mL}$ SonoVue ${ }^{\circledR}$ followed by a $5-\mathrm{mL}$ intravenous

254 injection of saline. Time between each injection $(\delta t)$ is 3.5 minutes. 
259 The primary measure for evaluating the effectiveness of the treatment was the amount

260 of cycles the patient could undergo. The more treatment cycles the patient underwent,

261 the longer the patient was considered healthy $[50,51]$. Furthermore, if the tumour size

262 was reduced substantially in accordance to the Response Evaluation Criteria in Solid

263 Tumours (RECIST) [52], the treatment modality was re-evaluated, e.g. transfer to

264 radiation therapy or surgery. This was considered a successful treatment.

265 Diagnostic ultrasound imaging was performed weekly assessing the tumour size. As

266 Computerised Tomography CT scans are considered the golden standard for

267 following tumour growth [53], every 8 weeks a CT scan was also performed to

268 validate the tumour size. This value was used to follow the tumour progression.

269 Positron Emission Tomography (PET) imaging was also performed at the start of the 270 treatment to assess the presence of metastasis.

271 Figure 6 shows the pancreatic adenocarcinoma in patient 5 prior to ultrasound and 272 microbubble treatment as seen by CT and PET imaging modalities. 


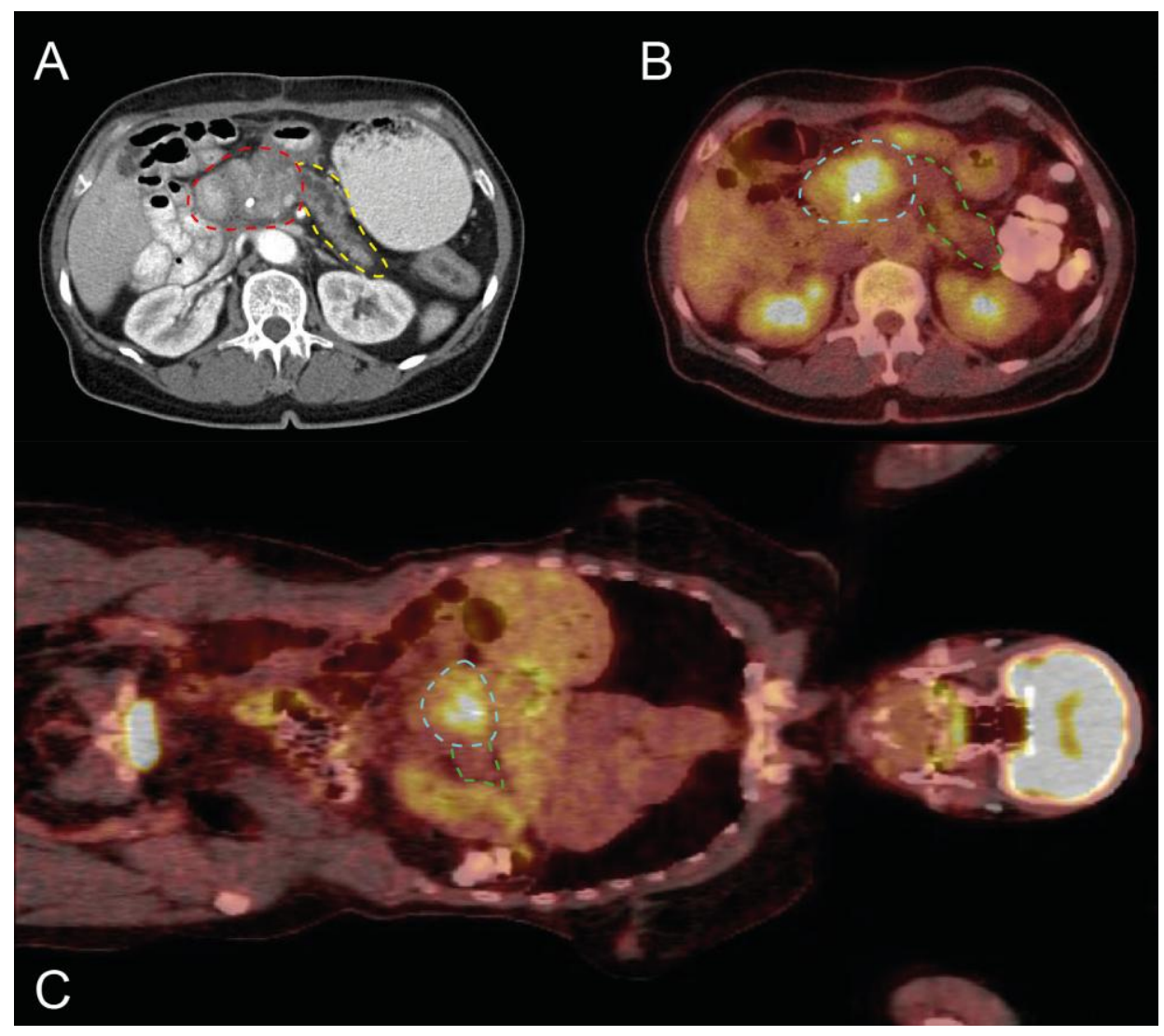

Figure 6. CT (Panel A) and PET (Panels B and C) images of patient 5 showing pancreatic adenocarcinoma prior to treatment. Panel A shows a CT scan in the transverse plane with the primary tumour in the head of the pancreas, and the pancreas indicated by the red and yellow dashed lines respectively. Panels B and C show PET scans in transverse and coronal plane respectively. The location of the tumour can be clearly identified by the brighter colour in the middle of the abdomen. In Panels $\mathrm{B}$ and $\mathrm{C}$, the tumour and pancreas are respectively indicated by the blue and green dashed lines. The pancreas tail is behind the large colon in panel C.

\section{Treatment group}

276 Patients with inoperable pancreatic cancer and fulfilled the inclusion criteria at the

277 Haukeland University Hospital, Bergen, Norway, who have volunteered to participate,

278 were included. The inclusion critera primarily stated that the patients must be $>18$ 
years of age, a diagnosis of inoperable pancreatic cancer, histologically verified,

280 locally advanced (stage II/III) or metastatic (stage IV) adenocarcinoma of the

281 pancreas, and must be ambulatory with an ECOG performance status between 0 and 2.

282 For this case report a total of five patients were recruited. Table 3 shows the

283 characteristics of the five patients enrolled in this pilot study prior to treatment in

284 addition to the start and end dates of the treatment for every patient.



285 Table 3: Patient characteristics prior to treatment. ND denotes non-discernable

286 values. Start and end date of treatment are also stated.

\section{Control group}

290 Taking into account the guidelines for gemcitabine treatment, it can be deduced that

291 the more treatment cycles the patient can undergo, the longer the patient can be 292 considered healthy; hence the more effective the treatment. Once the patient surpasses

293 a Level 2 in the ECOG performance status guidelines, they would no longer receive

294 treatment; this would accordingly define the end of the healthy and ambulatory

295 period. Our control group consisted of 80 patients from 2009-2011 with histology 
296 showing pancreatic adenocarcinoma (matching the same criteria as our patients).

297 These patients received the identical chemotherapy treatment (in accordance to

298 Gemzar guidelines [50]) at Haukeland University Hospital, Bergen, Norway. The

299 control treatments were also discontinued once they surpassed and ECOG

300 performance grade of 2 or their blood counts dropped below the chemotherapy

301 guidelines. Patients who received a different treatment were excluded from the control

302 group. The data was accessed through the internal hospital medical system. The same

303 anonymous data will be available on the Norwegian national cancer registry.

Ethical Considerations

307 All experiments were performed with approval from the regional ethics committee 308 under reference number 2011/1601/REK vest.

\section{$310 \quad$ Results and discussion}

311 The beam characterisation showed that the clinical scanner took into account the

312 attenuation of soft tissue when varying the focal depth. This allowed for a good

313 prediction of the ultrasound profile in-situ and easy manipulation of the ultrasound 314 intensity and positioning. The "active" area that we assume enhances the 315 chemotherapy effect was long and wide in all cases independent of depth, surpassing 316 the tumour size, allowing a maximum flexibility on treatment area. It has be assumed 317 that there are some fluctuations in the sound field pressures due to tissue property 318 variations, but this should not drastically change the sound field in our case, as 319 acoustic propagation was only though soft tissue. Taking into account the vast range 320 of ultrasound intensities used to induce sonoporation, as seen in literature, we assume 
321 that sonoporation may be occurring at lower or higher acoustic pressures independent

322 of the varying attenuation of tissue. A benefit of using a clinical probe is also that due

323 to the synthetic aperture, objects obscuring the field of view do not affect the beam

324 formation in other areas; hence we can predict the ultrasound dose delivered to our

325 target area.

326 The image generated using our customised treatment settings allowed easy

327 identification of both microbubbles and tumours. Figure 4A shows clear signs of

328 microbubble presence in the tumour vasculature and surrounding tissue. Figure 4B

329 shows the dimensions of a pancreatic tumour indicating the ease of detecting and

330 aligning the probe to the tumour using the modified settings.

331 Figure 7 shows the normalised perfusion curve where the arrows indicate the contrast

332 injection time, as measured by the clinical scanner during the first 13 minutes of

333 ultrasound and microbubble treatment. A pseudo-sinusoidal perfusion curve can be

334 seen. Throughout the whole treatment we can see that there are always microbubbles

335 present. By using this pseudo-continuous method we can ensure that there are always

336 microbubbles present without the added complexity of continuous infusion

337 equipment. 


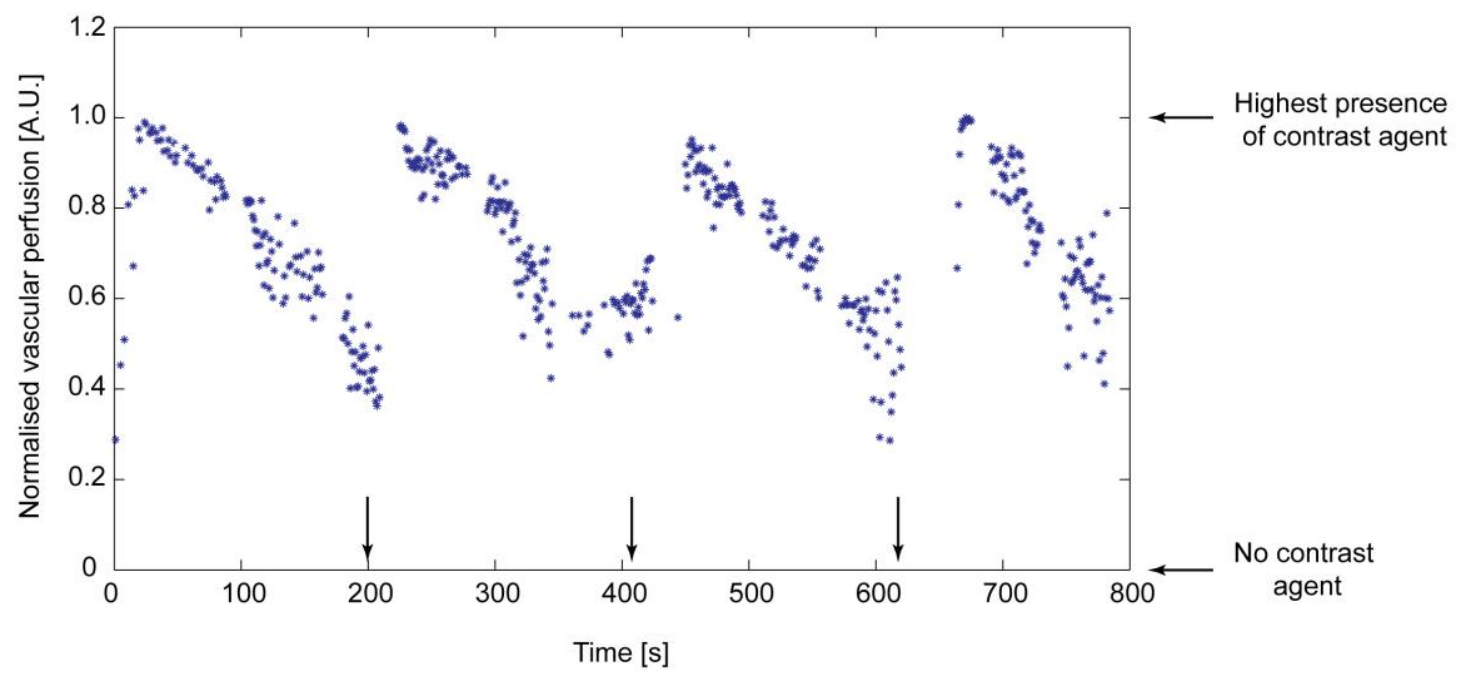

$340 \quad$ Figure 7: Normalised microbubble presence in tumour locality during the first $800 \mathrm{~s}$

341 of treatment. Arrows indicate contrast injection time.

343 Our control group, treated with the same chemotherapeutic protocol, received an 344 average of $9 \pm 6$ treatment cycles. To date all patients participating in this trial have 345 already surpassed this indicating the potential benefit of our combined treatment on a 346 clinical scale with minimal changes to chemotherapy protocols. The patients enrolled 347 in this clinical pilot study received and average of $16 \pm 7$ treatment cycles.

348 Figure 8 and Table 3 show the effect of our combined treatment on the tumour size. 349 After 8 weeks two patients showed a tumour diameter reduction. Patient 1 had a 350 temporary tumour reduction from $4.0 \mathrm{~cm}$ to $3.1 \mathrm{~cm}$. The next CT image was taken 24 351 weeks later and showed a growth to $4.6 \mathrm{~cm}$; an increase of $15 \%$ from the original 352 tumour size after 32 weeks of treatment. In patient 2, the treatment resulted in a 353 continuous tumour reduction over 16 weeks, a very rare response from chemotherapy 354 alone. As a result of his increased health, after 10 treatment cycles, he was removed 355 from the clinical trial to undergo radiation therapy. As this patient was removed from 356 the trial due to the success of the treatment, a lower number of total and average 357 treatments was seen, reducing the apparent effectiveness of the treatment as a whole. 
358 It should be noted that none of the patients in the control group stopped treatment due

359 to its success but on the contrary, due to their deterioration.

360 Two patients showed slow tumour growth from the $8^{\text {th }}$ week onwards (patient 3 and 361 patient 4). Patient 5 also had a biopsy verified primary tumour in the pancreas. This 362 was surgically removed but re-occurred with a small tumour in the operation sight and 363 a large metastasis. This indicated that the tumour was at a late stage of development 364 hence a limited response could be expected from the chemotherapeutic. Nevertheless, 365 this patient was also able to receive 11 cycles of treatment.

366 As pancreatic cancer is such an aggressive form of cancer it is very uncommon to see 367 any decrease in tumour growth from chemotherapy. Our aim was to improve quality 368 of life, to extend the healthy period of life, and conclusively extend the patients 369 survival. If the patient was "healthy" enough (well-defined state in both groups, 370 ECOG performance status 0-2 [51]), they would be able to receive treatment for a 371 longer period. In fact, as long they are ambulatory and capable of all self-care they are 372 able to receive the treatment. Seeing a decrease in the primary tumour size was an 373 added benefit to the increased number of treatment cycles and thereby the anticipated 374 survival. 


\begin{tabular}{|c|c|c|c|c|c|c|}
\hline \multirow{2}{*}{ Patient } & \multicolumn{5}{|c|}{ Maximum tumour diameter (cm) } & Total \\
\cline { 2 - 7 } & Inclusion day & Week 8 & Week 16 & Week 24 & Week 32 & of \\
& & & & & & cycles \\
\hline 1 & 4.0 & 3.1 & - & - & 4.6 & 27 \\
\hline 2 & 4.9 & 3.9 & 3.6 & - & - & 10 \\
\hline 3 & 1.5 & 3.0 & 3.5 & - & - & 11 \\
\hline 4 & 2.2 & - & 2.3 & 2.9 & 3.4 & 16 \\
\hline 5 & 4.0 & - & 5.5 & 6.2 & - & 16 \\
\hline
\end{tabular}

Table 4: Maximum tumour diameter as measured from CT images. Empty values denote skipped CT scans.

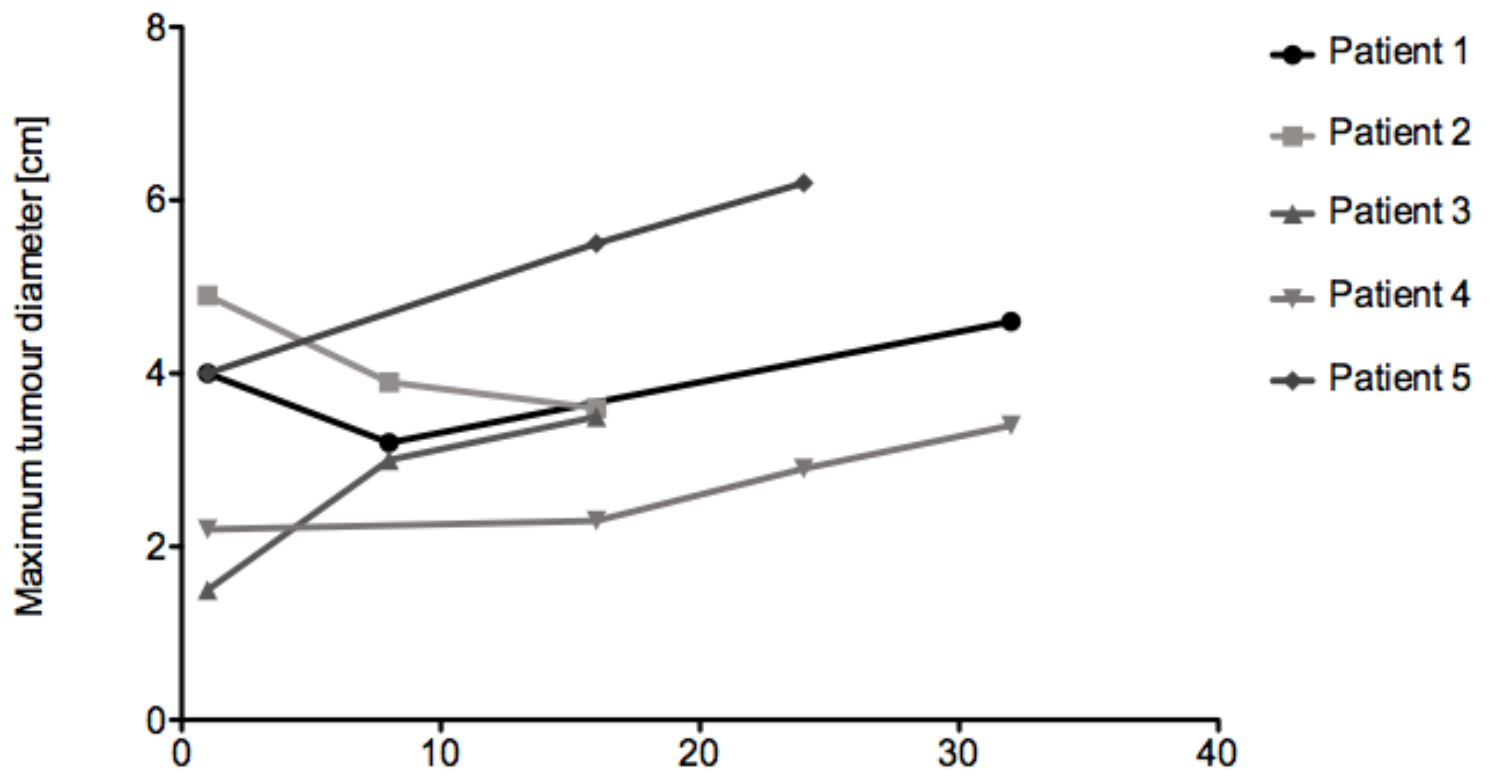

Time [Weeks]

Figure 8: Change in tumour diameter over time measured from CT images in patients

386 with pancreatic malignancy. 
388 The addition of the sonoporation procedure following the standard chemotherapeutic 389 protocol did not add any discomfort to the patients. All patients were very relaxed

390 during the treatment to a state where they could comfortably sleep throughout the 391 whole treatment.

392 In this study we also aimed to show that it is possible to induce sonoporation in the 393 clinic using existing commercial equipment, whilst fitting in the current safety 394 regulations for the use of diagnostic ultrasound. In our previous work we showed that 395 a duty cycle of $40 \%$ was ideal for sonoporation [7, 23]. Here we are using a duty 396 cycle of $1 \%$; hence expecting a small effect of sonoporation. There are many ways to 397 improve this method of therapy such as by increasing the duty cycle from $1 \%$ to $40 \%$ 398 and introducing targeted microbubbles that could attach to specific cancer cells [40]. 399 The efficacy of our combined treatment should be compared to the efficacy of the 400 current golden standard, the chemotherapeutic gemcitabine alone, where the viability 401 of the patient has been extended by approximately 1 month [3, 4].

\section{Conclusion}

403 Using a clinical diagnostic scanner for therapeutic purposes allows accurate acoustic 404 field alignment ensuring that the desired ultrasound dose reaches the target area. This 405 configuration allows simultaneous visualisation of the microbubbles present whilst 406 treating the pancreatic tumour. In this pilot study, we saw an extended treatment 407 period when comparing to the control group. Furthermore, we did not notice any 408 adverse side effects. Combined ultrasound, microbubble and chemotherapeutic 409 treatment could pave the way for a novel enhanced drug delivery pathways. 


\section{Acknowledgements}

414 This study has been supported by funds from the Norwegian Cancer Society (NCS)

415 and MedViz (http://medviz.uib.no/), an interdisciplinary research cluster from

416 Haukeland University Hospital, University of Bergen and Christian Michelsen

417 Research AS. We would like to thank Dr. Martin Biermann, Dr. Tormod Bjårnes, Dr.

418 Bjørn Tore Gjertsen, Dr. Anders Molven and Dr. Halfdan Sørbye for their support

419 throughout this project.

420

421 
[1] World Health Organization. (2012). World Health Statistics. Available:

[4] J. P. Neoptolemos, D. D. Stocken, H. Friess, C. Bassi, J. A. Dunn, H. Hickey, H. Beger, L. Fernandez-Cruz, C. Dervenis, F. Lacaine, M. Falconi, P. Pederzoli, A. Pap, D. Spooner, D. J. Kerr, M. W. Buchler, and C. European Study Group for Pancreatic, "A randomized trial of chemoradiotherapy and chemotherapy after resection of pancreatic cancer," $N$ Engl J Med, vol. 350, pp. 1200-1210, (2004).

[5] S. Bao, B. D. Thrall, and D. L. Miller, "Transfection of a reporter plasmid into cultured cells by sonoporation in vitro," Ultrasound Med. Biol., vol. 23, pp. 953-959, (1997).

[6] A. van Wamel, K. Kooiman, M. Harteveld, M. Emmer, F. J. ten Cate, M. Versluis, and N. de Jong, "Vibrating microbubbles poking individual cells: drug transfer into cells via sonoporation," J. Control. Release, vol. 112, pp. 149-155, (2006).

[7] A. Delalande, S. Kotopoulis, T. Rovers, C. Pichon, and M. Postema, "Sonoporation at a low mechanical index," Bub. Sci. Eng. Tech., vol. 3, pp. 311, (2011).

[8] M. Postema, S. Kotopoulis, A. Delalande, and O. H. Gilja, "Sonoporation: Why microbubbles create pores," Ultraschall in Med., vol. 33, pp. 97-98, (2012).

[9] N. G. Lee, J. L. Berry, T. C. Lee, A. T. Wang, S. Honowitz, A. L. Murphree, N. Varshney, D. R. Hinton, and A. A. Fawzi, "Sonoporation enhances chemotherapeutic efficacy in retinoblastoma cells in vitro," Invest Ophthalmol Vis Sci, vol. 52, pp. 3868-3873, (2011).

[10] C. X. Deng, F. Sieling, H. Pan, and J. Cui, "Ultrasound-induced cell membrane porosity," Ultrasound Med Biol, vol. 30, pp. 519-526, (2004).

[11] R. K. Schlicher, H. Radhakrishna, T. P. Tolentino, R. P. Apkarian, V. Zarnitsyn, and M. R. Prausnitz, "Mechanism of intracellular delivery by acoustic cavitation," Ultrasound Med Biol, vol. 32, pp. 915-924, (2006).

[12] Y. Z. Zhao, Y. K. Luo, C. T. Lu, J. F. Xu, J. Tang, M. Zhang, Y. Zhang, and H. D. Liang, "Phospholipids-based microbubbles sonoporation pore size and reseal of cell membrane cultured in vitro," J Drug Target, vol. 16, pp. 18-25, (2008).

[13] M. Postema and O. H. Gilja, "Ultrasound-directed drug delivery," Curr. Pharm. Biotechnol., vol. 8, pp. 355-361, (2007). 
[14] M. Postema, O. H. Gilja, and A. van Wamel, "CEUS and sonoporation," in Fundamentals of Medical Ultrasonics, M. Postema, Ed., London: Spon press, 2011, pp. 205-217.

[15] M. Postema, S. Kotopoulis, A. Delalande, and O. H. Gilja, "Ultrasound-guided delivery and sonoporation," in Ultrasound in Gastroenterology. 10-years aniversary of National Center for Ultrasound in Gastroenterology, 2011, pp. 57-59.

[16] J. M. Escoffre, A. Novell, J. Piron, A. Zeghimi, A. Doinikov, and A. Bouakaz, "Microbubble attenuation and destruction: are they involved in sonoporation efficiency?," IEEE Trans Ultrason Ferroelectr Freq Control, vol. 60, pp. 4652, (2013).

[17] D. L. Miller and C. Dou, "Membrane damage thresholds for 1- to $10-\mathrm{MHz}$ pulsed ultrasound exposure of phagocytic cells loaded with contrast agent gas bodies in vitro," Ultrasound Med. Biol., vol. 30, pp. 973-977, (2004).

[18] D. L. Miller and C. Dou, "Membrane damage thresholds for pulsed or continuous ultrasound in phagocytic cells loaded with contrast agent gas bodies," Ultrasound Med. Biol., vol. 30, pp. 405-411, (2004).

[19] D. L. Miller, C. Dou, and J. Song, "DNA transfer and cell killing in epidermoid cells by diagnostic ultrasound activation of contrast agent gas bodies in vitro," Ultrasound Med. Biol., vol. 29, pp. 601-607, (2003).

[20] D. L. Miller and J. Quddus, "Sonoporation of monolayer cells by diagnostic ultrasound activation of contrast-agent gas bodies," Ultrasound Med. Biol., vol. 26, pp. 661-667, (2000).

[21] M. W. Miller, "Gene transfection and drug delivery," Ultrasound Med. Biol., vol. 26, pp. S59-S62, (2000).

[22] K. Kooiman, M. Harteveld, A. F. W. van der Steen, and N. de Jong, "Sonoporation of endothelial cells by vibrating targeted microbubbles," $J$. Control Release, vol. 154, pp. 35-41, (2011).

[23] A. Delalande, A. Bouakaz, G. Renault, F. Tabareau, S. Kotopoulis, P. Midoux, B. Arbeille, R. Uzbekov, S. Chakravarti, M. Postema, and C. Pichon, "Ultrasound and microbubble-assisted gene delivery in Achilles tendons: long lasting gene expression and restoration of fibromodulin KO phenotype," $J$ Control Release, vol. 156, pp. 223-230, (2011).

[24] S. M. Nejad, S. H. R. Hosseini, H. Akiyama, and K. Tachibana, "Optical observation of cell sonoporation with low intensity ultrasound," Biochemical and Biophysical Research Communications, vol. 413, pp. 218-223, (2011).

[25] F. Yang, N. Gu, D. Chen, X. Xi, D. Zhang, Y. Li, and J. Wu, "Experimental study on cell self-sealing during sonoporation," J Control Release, vol. 131, pp. 205-210, (2008).

[26] Y. Qiu, C. Zhang, J. Tu, and D. Zhang, "Microbubble-induced sonoporation involved in ultrasound-mediated DNA transfection in vitro at low acoustic pressures," Journal of Biomechanics, vol. 45, pp. 1339-1345, (2012).

[27] M. Matsuo, K. Yamaguchi, L. B. Feril, Jr., H. Endo, K. Ogawa, K. Tachibana, and J. Nakayama, "Synergistic inhibition of malignant melanoma proliferation by melphalan combined with ultrasound and microbubbles," Ultrason Sonochem, vol. 18, pp. 1218-1224, (2011).

[28] N. Lamanauskas, A. Novell, J. M. Escoffre, M. Venslauskas, S. Satkauskas, and A. Bouakaz, "Bleomycin delivery into cancer cells in vitro with ultrasound and SonoVue ${ }^{\circledR}$ or BR14® microbubbles.," J Drug Target, vol. 1, p. [Epub ahead of print], (2013). 
[29] Y. Watanabe, A. Aoi, S. Horie, N. Tomita, S. Mori, H. Morikawa, Y. Matsumura, G. Vassaux, and T. Kodama, "Low-intensity ultrasound and microbubbles enhance the antitumor effect of cisplatin," Cancer Sci, vol. 99, pp. 2525-2531, (2008).

[30] J. Wu, J. Pepe, and M. Rincon, "Sonoporation, anti-cancer drug and antibody delivery using ultrasound," Ultrasonics, vol. 44, pp. E21-E25, (2006).

[31] C. Y. Lai, C. H. Wu, C. C.C., and P. C. Li, "Quantitative relations of acoustic inertial cavitation with sonoporation and cell viability," Ultrasound Med. Biol., vol. 32, pp. 1931-1941, (2006).

[32] D. M. Hallow, A. D. Mahajan, T. E. McCutchen, and M. R. Prausnitz, "Measurement and correlation of acoustic cavitation with cellular bioeffects," Ultrasound Med. Biol., vol. 32, pp. 1111-1122, (2006).

[33] M. M. Forbes, R. L. Steinberg, and W. D. O'Brien, "Examination of Inertial Cavitation of Optison in Producing Sonoporation of Chinese Hamster Ovary Cells," Ultrasound in Medicine and Biology, vol. 34, pp. 2009-2018, (2008).

[34] J. L. Tlaxca, C. R. Anderson, A. L. Klibanov, B. Lowrey, J. A. Hossack, J. S. Alexander, M. B. Lawrence, and J. J. Rychak, "Analysis of in vitro Transfection by Sonoporation Using Cationic and Neutral Microbubbles," Ultrasound Med Biol, vol. 36, pp. 1907-1918, (2010).

[35] C. D. Ohl and B. Wolfrum, "Detachment and sonoporation of adherent HeLacells by shock wave-induced cavitation," Biochimica Et Biophysica ActaGeneral Subjects, vol. 1624, pp. 131-138, (2003).

[36] D. L. Miller and C. Y. Dou, "Induction of Apoptosis in Sonoporation and Ultrasonic Gene Transfer," Ultrasound in Medicine and Biology, vol. 35, pp. 144-154, (2009).

[37] M. Postema and O. H. Gilja, "Jetting does not cause sonoporation," Biomed. Eng., vol. 55, pp. S19-S20, (2010).

[38] B. Geers, H. Dewitte, S. C. De Smedt, and I. Lentacker, "Crucial factors and emerging concepts in ultrasound-triggered drug delivery," J Control Release, vol. 164, pp. 248-255, (2012).

[39] F. G. Erchinger, G. Dimcevski, T. Engjom, and O. H. Gilja, "Transabdominal ultrasonography of the pancreas: basic and new aspects," Imaging Med., vol. 3, pp. 412-422, (2011).

[40] M. Postema and O. H. Gilja, "Contrast-enchanced and targeted ultrasound," World J. Gastroenterol., vol. 17, pp. 28-41, (2011).

[41] F. Piscaglia, C. Nolsoe, C. F. Dietrich, D. O. Cosgrove, O. H. Gilja, M. Bachmann Nielsen, T. Albrecht, L. Barozzi, M. Bertolotto, O. Catalano, M. Claudon, D. A. Clevert, J. M. Correas, M. D'Onofrio, F. M. Drudi, J. Eyding, M. Giovannini, M. Hocke, A. Ignee, E. M. Jung, A. S. Klauser, N. Lassau, E. Leen, G. Mathis, A. Saftoiu, G. Seidel, P. S. Sidhu, G. ter Haar, D. Timmerman, and H. P. Weskott, "The EFSUMB Guidelines and Recommendations on the Clinical Practice of Contrast Enhanced Ultrasound (CEUS): update 2011 on non-hepatic applications," Ultraschall in Med., vol. 33, pp. 33-59, (2012).

[42] M. Postema, Fundamentals of medical ultrasonics. Milton Park, Abingdon, Oxon ; New York: Spon Press, 2011.

[43] B. Gerold, S. Kotopoulis, M. C., D. McGloin, M. Postema, and P. Prentice, "Laser-nucleated acoustic cavitation in focused ultrasound," Rev. Sci. Instrum., vol. 82, p. 044908, (2011). 
[44] U.S. Department of Health and Human Services. Food and Drug Administration, "Information for Manufacturers Seeking Marketing Clearance of Diagnostic Ultrasound Systems and Transducers," 2008.

[45] International Electrotechnical Commision, "Ultrasonics - Hydrophones - Part 2: Calibration for ultrasonic fields up to $40 \mathrm{MHz}, " 2013$.

[46] J. A. Jensen, S. I. Nikolov, K. L. Gammelmark, and M. H. Pedersen, "Synthetic aperture ultrasound imaging," Ultrasonics, vol. 44 Suppl 1, pp. e515, (2006).

[47] E. Quaia, "Microbubble ultrasound contrast agents: an update," European Radiology, vol. 17, pp. 1995-2008, (2007).

[48] British Medical Ultrasound Society, Guidelines for the safe use of diagnostic ultrasound equipment, 2000.

[49] S. B. Barnett, G. R. Ter Haar, M. C. Ziskin, H. D. Rott, F. A. Duck, and K. Maeda, "International recommendations and guidelines for the safe use of diagnostic ultrasound in medicine," Ultrasound Med Biol, vol. 26, pp. 355-366, (2000).

[50] Eli Lilly and Company. (2010). Highlights of prescribing information Gemzar. Available: http://pi.lilly.com/us/gemzar.pdf

[51] M. M. Oken, R. H. Creech, D. C. Tormey, J. Horton, T. E. Davis, E. T. Mcfadden, and P. P. Carbone, "Toxicity and Response Criteria of the EasternCooperative-Oncology-Group," American Journal of Clinical OncologyCancer Clinical Trials, vol. 5, pp. 649-655, (1982).

[52] E. A. Eisenhauer, P. Therasse, J. Bogaerts, L. H. Schwartz, D. Sargent, R. Ford, J. Dancey, S. Arbuck, S. Gwyther, M. Mooney, L. Rubinstein, L. Shankar, L. Dodd, R. Kaplan, D. Lacombe, and J. Verweij, "New response evaluation criteria in solid tumours: Revised RECIST guideline (version 1.1)," European Journal of Cancer, vol. 45, pp. 228-247, (2009).

[53] S. A. Sohaib, B. Turner, J. A. Hanson, M. Farquharson, R. T. Oliver, and R. H. Reznek, "CT assessment of tumour response to treatment: comparison of linear, cross-sectional and volumetric measures of tumour size," $\mathrm{Br} \mathrm{J}$ Radiol, vol. 73, pp. 1178-1184, (2000). 